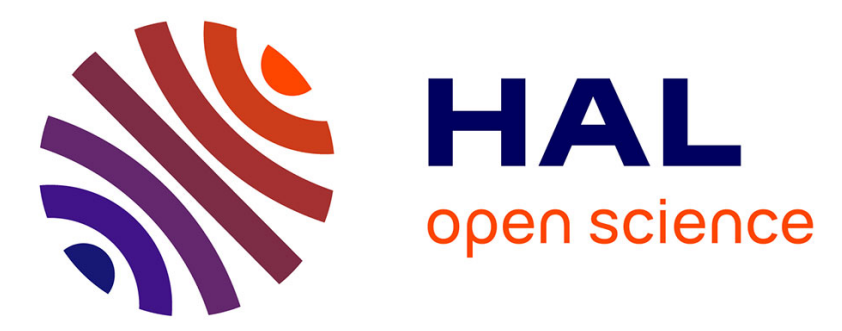

\title{
Haldane phases with ultracold fermionic atoms in double-well optical lattices
}

P. Fromholz, Sylvain Capponi, P. Lecheminant, David Papoular, K. Totsuka

\section{To cite this version:}

P. Fromholz, Sylvain Capponi, P. Lecheminant, David Papoular, K. Totsuka. Haldane phases with ultracold fermionic atoms in double-well optical lattices. Phys.Rev.B, 2019, 99 (5), pp.054414. 10.1103/PhysRevB.99.054414 . hal-02058539

\section{HAL Id: hal-02058539 \\ https://hal.science/hal-02058539}

Submitted on 21 Nov 2019

HAL is a multi-disciplinary open access archive for the deposit and dissemination of scientific research documents, whether they are published or not. The documents may come from teaching and research institutions in France or abroad, or from public or private research centers.
L'archive ouverte pluridisciplinaire HAL, est destinée au dépôt et à la diffusion de documents scientifiques de niveau recherche, publiés ou non, émanant des établissements d'enseignement et de recherche français ou étrangers, des laboratoires publics ou privés. 


\title{
Haldane phases with ultracold fermionic atoms in double-well optical lattices
}

\author{
P. Fromholz, ${ }^{1}$ S. Capponi, ${ }^{2}$ P. Lecheminant, ${ }^{1}$ D. J. Papoular, ${ }^{1}$ and K. Totsuka ${ }^{3}$ \\ ${ }^{1}$ Laboratoire de Physique Théorique et Modélisation, CNRS UMR 8089, Université de Cergy-Pontoise, Site de Saint-Martin, \\ F-95300 Cergy-Pontoise Cedex, France \\ ${ }^{2}$ Laboratoire de Physique Théorique, CNRS UMR 5152, Université Paul Sabatier, F-31062 Toulouse, France \\ ${ }^{3}$ Yukawa Institute for Theoretical Physics, Kyoto University, Kitashirakawa Oiwake-Cho, Kyoto 606-8502, Japan
}

(Received 12 October 2017; revised manuscript received 13 July 2018; published 15 February 2019)

\begin{abstract}
We propose to realize one-dimensional topological phases protected by $\mathrm{SU}(N)$ symmetry using alkali or alkaline-earth atoms loaded into a bichromatic optical lattice. We derive a realistic model for this system and investigate it theoretically. Depending on the parity of $N$, two different classes of symmetry-protected topological (SPT) phases are stabilized at half-filling for physical parameters of the model. For even $N$, the celebrated spin-1 Haldane phase and its generalization to $\mathrm{SU}(N)$ are obtained with no local symmetry breaking. In stark contrast, at least for $N=3$, a new class of SPT phases, dubbed chiral Haldane phases, that spontaneously break inversion symmetry, emerges with a twofold ground-state degeneracy. The latter ground states with open-boundary conditions are characterized by different left and right boundary spins, which are related by conjugation. Our results show that topological phases are within close reach of the latest experiments on cold fermions in optical lattices.
\end{abstract}

DOI: 10.1103/PhysRevB.99.054414

\section{INTRODUCTION}

Symmetry-protected topological (SPT) phases have recently attracted lots of attention. These new quantum phases exhibit short-range entanglement and possess only conventional gapped excitations in the bulk while hosting nontrivial symmetry-protected surface states [1,2]. A paradigmatic example of one-dimensional (1D) bosonic SPT phases is the Haldane phase of the spin-1 antiferromagnetic spin chain [3]. While the bulk of this phase looks ordinary, nontrivial spin$1 / 2$ edge states appear for an open-boundary condition [4] or when the chain is cut by doping impurities [5]. This phase is protected by the $\mathrm{SO}(3)$ symmetry underlying the Heisenberg model, and more generally by at least one of the three discrete symmetries: the dihedral group of $\pi$-rotations along the $x, y, z$ axes, time-reversal or inversion symmetries [6,7].

A fairly complete understanding of 1D bosonic SPT phases has been obtained through group cohomology, matrix-product states, entanglement spectroscopy, and field-theoretical arguments [8-12]. The possible 1D SPT phases associated with a given protecting symmetry $G$ are classified by its projective representations, i.e., the second cohomology group $\mathcal{H}^{2}(G, \mathrm{U}(1))$ : in the presence of $\mathrm{SO}(3)$ symmetry, there is a $\mathbb{Z}_{2}$ classification and the Haldane phase is the only SPT phase whose edge states obey a nontrivial projective representation $[6,7]$.

Richer SPT phases can be obtained when $G$ is a more general Lie group. For instance, the group $\mathrm{SU}(N)$ leads to a $\mathbb{Z}_{N}$ classification predicting $N-1$ nontrivial SPT phases [13] protected by $\mathrm{SU}(N)$ [PSU $(N)$, more precisely [14]] or by its discrete subgroup $\mathbb{Z}_{N} \times \mathbb{Z}_{N}[15,16]$. Their edge states are labeled by the inequivalent projective representations of $\mathrm{SU}(N)$, which are specified by $\mathbb{Z}_{N}$ quantum numbers $n_{\text {top }}=n_{\mathrm{Y}}(\bmod N)$, with $n_{\mathrm{Y}}$ being the number of boxes in the Young diagram corresponding to the representation of the boundary spins $[13,17]$ [readers unfamiliar with $\mathrm{SU}(N)$ and its representation are referred to Sec. I of [18]). In stark contrast to the $N=2$ case, i.e., $G=\mathrm{SO}(3)$, where all the projective representations are self-conjugate, the left and right edge states of the $\mathrm{SU}(N)$ SPT phases with $N>2$ might belong to different projective representations that are related by conjugation. This leads to an interesting class of SPT phases, dubbed chiral Haldane $(\chi \mathrm{H})$. These phases necessitate broken inversion symmetry (whether explicit or spontaneous) [18] and, when it is broken spontaneously, they exist in pairs; in one phase, the left and right edge states transform, respectively, in the $\mathrm{SU}(N)$ representation $\mathcal{R}$ and its conjugate $\overline{\mathcal{R}}$, and vice versa in the other [19-23]. In the following, we label the SPT phases by the number of boxes in the Young diagrams as $\left(n_{\mathrm{Y}}(\mathcal{R}), n_{\mathrm{Y}}(\overline{\mathcal{R}})\right)(\bmod N)$. In reflection-symmetric systems, the two topological ground states $\left(n_{\mathrm{Y}}(\mathcal{R}), n_{\mathrm{Y}}(\overline{\mathcal{R}})\right)$ and $\left(n_{\mathrm{Y}}(\overline{\mathcal{R}}), n_{\mathrm{Y}}(\mathcal{R})\right)$ are degenerate.

In this paper, we propose an implementation of the Haldane phase $(N=2)$ and its generalizations to even- $N$, as well as the $\chi \mathrm{H}$ phases for $N=3$, with half-filled ultracold fermions loaded into 1D double-well optical lattices. These clean and controllable systems offer an ideal framework for realizing the SPT phases, which require precise symmetries. The $N=2$ case may be realized using the two lowest hyperfine states of ${ }^{6} \mathrm{Li}$. Larger values of $N$ may be explored experimentally using ${ }^{87} \mathrm{Sr}$ or ${ }^{173} \mathrm{Yb}$ atoms in their ${ }^{1} S_{0}$ ground states, which possess $\mathrm{SU}(N)$-symmetry $(N \leqslant 10)$ [24-30]. By means of complementary strong-coupling and numerical techniques, we show that, for all even $N \geqslant 2$ and (at least) $N=3$, fully gapped featureless Mott-insulating phases show up in the phase diagram of the underlying lattice fermion models with repulsive interactions. The phases occurring for even- $N$ are identified as the Haldane phase $(N=2)$ or its generalization 


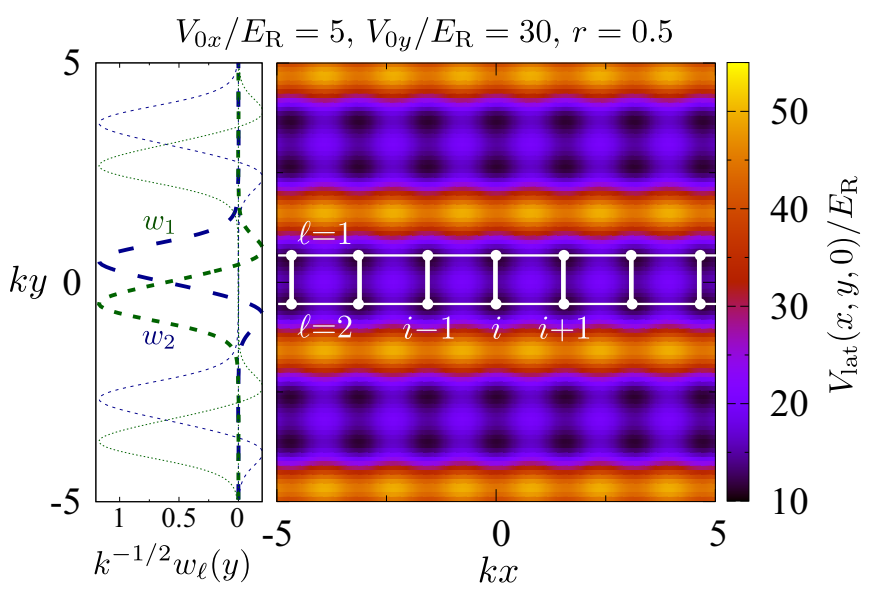

FIG. 1. The potential $V_{\text {lat }}$ in the $x y$ plane (right panel): three double-well ladder systems are visible. The two independent Wannier functions $w_{1}(y)$ and $w_{2}(y)$ along $y$ (left, green and blue) are centered on the two chains $(\ell=1,2)$. Lengths and energies are, respectively, expressed in units of the reduced wavelength $1 / k$ and the recoil energy $E_{R}=\hbar^{2} k^{2} / 2 m$. This optical potential yields $t_{\perp} / t=$ 2.9 and $V / U=0.086$.

$(N \geqslant 4)$. On the other hand, for odd $N$ (at least for $N=$ 3 ), we find that $\chi \mathrm{H}$ phases emerge breaking the inversion symmetry spontaneously. These SPT phases are stabilized for realistic parameters of the model, paving the way toward their experimental investigation for $N \leqslant 10$.

\section{MODEL}

We consider ultracold (alkali, alkaline-earth, or ytterbium) fermions with $\mathrm{SU}(N)$ symmetry, trapped inside the following potential representing a three-dimensional array of double wells (see Fig. 1):

$$
\begin{aligned}
V_{\text {lat }}(x, y, z)= & V_{0 y}\left[\sin ^{2}(k y)+r \cos ^{2}(2 k y)\right] \\
& +V_{0 x} \sin ^{2}(2 k x)+V_{0 z} \sin ^{2}(2 k z),
\end{aligned}
$$

where $1 / k$ denotes the reduced wavelength and $r$ is a tunable parameter. This potential can be realized optically, using a bichromatic lattice [31] or exploiting interference patterns involving two differently polarized light beams [32]. Choosing sufficiently large values of $V_{0 y}$ and $V_{0 z}$, we obtain a single 1D two-leg ladder whose legs ( $\ell=1$ or 2 ) and rungs (labeled $i$ ) are, respectively, parallel to the $x$ and $y$ axes.

We restrict our analysis to the lowest bands in the $x$ and $z$ directions. In the $y$-direction, we keep the two lowest bands to resolve the two minima of each double well. This leads to the following lattice model:

$$
\begin{aligned}
\mathcal{H}_{0}= & -t \sum_{i, \ell} \sum_{\alpha=1}^{N}\left(c_{\ell \alpha, i+1}^{\dagger} c_{\ell \alpha, i}+\text { H.c. }\right)-\mu \sum_{i} n_{i} \\
& -t_{\perp} \sum_{i} \sum_{\alpha=1}^{N}\left(c_{1 \alpha, i}^{\dagger} c_{2 \alpha, i}+\text { H.c. }\right),
\end{aligned}
$$

where $c_{\ell \alpha, i}^{\dagger}$ creates a fermion in the nuclear-spin state $\alpha(=$ $1, \ldots, N)$ on the leg $\ell$ and the rung $i$. In Eq. (2), the total density operator on the rung $i$ is $n_{i}=\sum_{\ell \alpha} c_{\ell \alpha, i}^{\dagger} c_{\ell \alpha, i}=\sum_{\ell \alpha} n_{\ell \alpha, i}$.
The tunneling amplitudes $t$ along a leg and $t_{\perp}$ along a rung may be different. We now account for $\mathrm{SU}(N)$-symmetric two-body interactions modeled by the contact Hamiltonian $g \sum_{\alpha \neq \beta} \int d^{3} r n_{\alpha}(\boldsymbol{r}) n_{\beta}(\boldsymbol{r})$, where $n_{\alpha}(\boldsymbol{r})$ is the density operator for fermions in the state $\alpha$ [24-26]. Retaining the same bands as in Eq. (2), we obtain the interaction Hamiltonian:

$$
\begin{aligned}
\mathcal{H}_{\text {int }}= & \frac{U}{2} \sum_{i} \sum_{\ell=1}^{2} \sum_{\alpha \neq \beta} n_{\ell \alpha, i} n_{\ell \beta, i} \\
& +V \sum_{i} \sum_{\alpha \neq \beta}\left\{n_{1 \alpha, i} n_{2 \beta, i}+c_{1 \alpha, i}^{\dagger} c_{2 \beta, i}^{\dagger} c_{1 \beta, i} c_{2 \alpha, i}\right. \\
& \left.+\frac{1}{2}\left(c_{1 \alpha, i}^{\dagger} c_{1 \beta, i}^{\dagger} c_{2 \beta, i} c_{2 \alpha, i}+\text { H.c. }\right)\right\},
\end{aligned}
$$

where $U$ is the on-site interaction, and $V$ encodes the off-site interaction between the two sites on a given rung. There are three types of off-site processes: (i) density-density interaction, (ii) spin-exchange interaction, and (iii) pair-hopping of fermions with different spins from one leg to the other. Hence, Eq. (3) can be viewed as a generalized two-leg fermionic $\mathrm{SU}(N)$ ladder model with pair-hopping processes. The coefficients $t, t_{\perp}, U$, and $V$ characterizing the lattice model $\mathcal{H}=\mathcal{H}_{0}+\mathcal{H}_{\text {int }}$ are determined by the Wannier functions [33] that are calculated numerically for $V_{\text {lat }}(\mathbf{r})$ as in Ref. [34]. Along the rung direction $y$, we choose the Wannier functions $w_{1}(y)$ and $w_{2}(y)$ to be real and localized on the legs $\ell=1$ and 2 , respectively (see Fig. 1). The orthogonality of the Wannier functions necessitates that $w_{1}(y)$ and $w_{2}(y)$ have a finite extent around their center with changing signs. The coefficient $V$ is proportional to $g \int d y w_{1}^{2} w_{2}^{2}$, and it is finite because of a nonzero overlap between the positive functions $w_{1}^{2}$ and $w_{2}^{2}$. Besides the above three interactions, density-assisted hopping terms [35], proportional to the integral $g \int d y w_{1} w_{2}^{3}$, are also present. However, now the sign change of the Wannier functions strongly suppresses the integral, so that we can safely drop them in Eq. (3) [36]. The ratios $t_{\perp} / t$ and $V / U$ are fixed by the optical potential $V_{\text {lat }}(x, y, z): t_{\perp} / t$ can be tuned from 1 to a few units by varying the parameter $r$ in Eq. (1) [37], whereas $V / U$ is of the order of $10^{-1}$. The ratio $U / t$ can be tuned using a magnetic Feshbach resonance $[38,39]$ or an optical Feshbach resonance for alkaline-earth atoms [40,41].

\section{STRONG-COUPLING ANALYSIS}

We now consider the atomic limit of the model (3) to look for SPT phases in the large- $U$ limit. Introducing the (anti)symmetric combinations $d_{1 \alpha, i}=\left(c_{1 \alpha, i}-c_{2 \alpha, i}\right) / \sqrt{2}$ and $d_{2 \alpha, i}=\left(c_{1 \alpha, i}+c_{2 \alpha, i}\right) / \sqrt{2}, \mathcal{H}$ reduces to the $p$-band model of Refs. [42-44] in an (effective) orbital magnetic field proportional to $t_{\perp}$ :

$$
\begin{aligned}
\mathcal{H}= & -t \sum_{i, \alpha} \sum_{m=1,2}\left(d_{m \alpha, i}^{\dagger} d_{m \alpha, i+1}+\text { H.c. }\right) \\
& -\left(\mu+\frac{U+V}{2}\right) \sum_{i} n_{i}+2 t_{\perp} \sum_{i} T_{i}^{z} \\
& +\frac{U+V}{4} \sum_{i} n_{i}^{2}+2 V \sum_{i}\left(T_{i}^{z}\right)^{2}+(U-V) \sum_{i}\left(T_{i}^{x}\right)^{2}
\end{aligned}
$$


where $T_{i}^{a}=\frac{1}{2} \sum_{m, n, \alpha} d_{m \alpha, i}^{\dagger} \sigma_{m n}^{a} d_{n \alpha, i}$ is the pseudospin operator for the orbital degrees of freedom and $\sigma^{a}(a=x, y, z)$ are the Pauli matrices. We restrict ourselves to half-filling (i.e., $N$ fermions per rung). The atomic-limit $\left(U, V, t_{\perp} \gg t\right)$ energy spectrum of the model (4) is readily obtained once the $\mathrm{SU}(N)$ and the pseudospin $(\mathbf{T})$ irreducible representations are known [18]. For even $N$, in most of the region $U>V>0$, the orbital pseudospin $\mathbf{T}$ is quenched to a singlet, while the $\mathrm{SU}(N)$ spin is maximized into a self-conjugate representation of $\mathrm{SU}(N)$ described by a Young diagram with two columns of lengths $N / 2$ [44]. To second order in $t$, the effective Hamiltonian is the $\mathrm{SU}(N)$ Heisenberg model [44]:

$$
\mathcal{H}_{\text {eff }}^{\text {(even) }}=J \sum_{i} \sum_{A=1}^{N^{2}-1} \mathcal{S}_{i+1}^{A} \mathcal{S}_{i}^{A},
$$

where $J=2 t^{2} /(U+V)$ is the spin-exchange constant, and $\mathcal{S}_{i}^{A}$ are the local $\mathrm{SU}(N)$ spin operators belonging to the selfconjugate representation mentioned above. For $N=2$, Eq. (5) reduces to the spin-1 Heisenberg chain, whose ground state is in the Haldane phase [3]. For generic even $N$, the ground-state properties of the model (5) have recently been investigated in detail in Refs. [17,44-47], where the ground state has been identified with an SU(N) SPT phase with $\mathbb{Z}_{N}$ quantum numbers $n_{\text {top }}=N / 2$ (with $n_{\text {top }}$ given by $n_{\mathrm{Y}}$ modulo $N$; see Sec. I B of [18]) characterized by edge states in the antisymmetric $(N / 2)$-tensor representation of $\mathrm{SU}(N)$. Remarkably, for odd $N$, the orbital degrees of freedom play a crucial role. Indeed, let us consider the $N=3$ case and start from $U=V$ and $t_{\perp}=0$, where each site of a rung is occupied either by $3(\square)$ or $\overline{\mathbf{3}}(\boxminus)$ in the atomic-limit ground state. Regarding $\mathbf{3}$ and $\overline{\mathbf{3}}$ as the two orbital states (e.g., up and down) and carrying out the second-order perturbation in $U-V$ and $t_{\perp}$, we obtain a spin-orbital effective Hamiltonian, which, when $U>V$, reduces to an $\mathrm{SU}(3)$ two-leg ladder with different spins (3 and $\overline{\mathbf{3}}$ ) on the two legs (Sec. II B in [48]). The couplings depend on the orbital part; after tracing it out, the system reduces to the two-leg ladder with diagonal interactions. We have shown numerically that the $\chi \mathrm{H}$ phase is stabilized only when finite diagonal interactions exist [48]. A relatively large $t_{\perp}(>0)$ polarizes the orbital pseudospins, and the diagonal couplings, which are crucial to the SPT phase, disappear. In fact, both the strong-coupling expansion assuming large $t_{\perp}$ and direct numerical simulations for large enough $t_{\perp}$ found only a featureless trivial phase, in agreement with the above scenario.

\section{NUMERICAL CALCULATIONS}

We mapped out the zero-temperature phase diagram of the model (4) at half-filling using density-matrix renormalizationgroup (DMRG) calculations [49]. We used open boundary conditions, keeping between 2000 and 4000 states depending on the parameters and sizes to keep a discarded weight below $10^{-5}$. We fix $t=1$ as the unit of energy and, instead of the full $\mathrm{SU}(N)$ symmetry, we have implemented the $\mathrm{U}(1)^{N}$ symmetry corresponding to the conservation of each species $\alpha=1, \ldots, N$. Starting with the simplest $N=2$ case, we reveal that the $\mathrm{SU}(N) \mathrm{SPT}$ phases, predicted in the

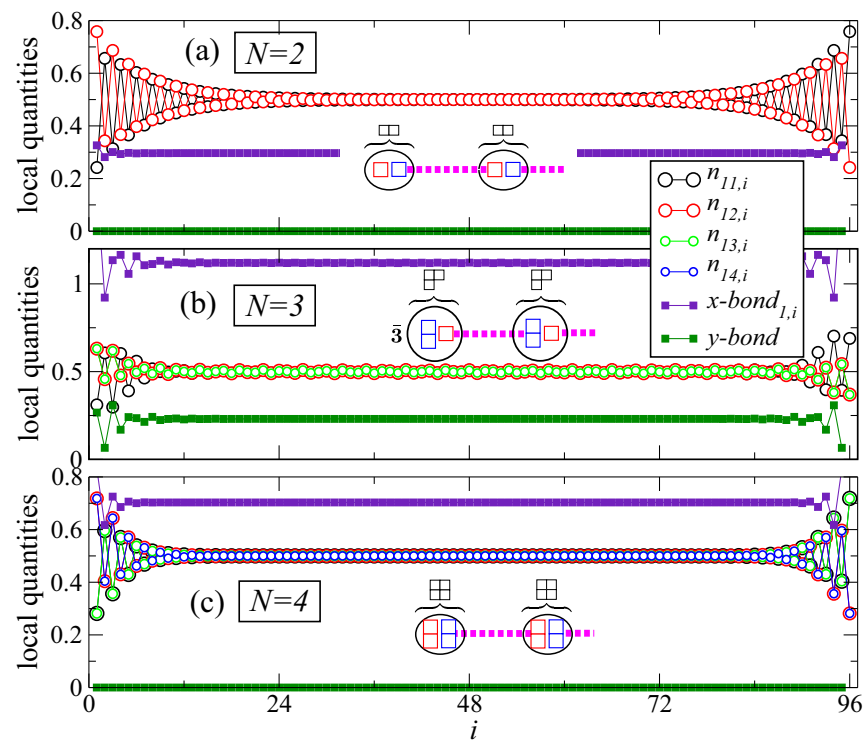

FIG. 2. Local densities and bond energies obtained by DMRG for a chain of length $L=96$ in the cases $N=2,3$, and 4, using $t_{\perp}=$ $1, U=12, V=4$. The densities and $x$-bond (i.e., rung) energies are found to be equal on both chains, and we show them for $\ell=1$. By summing up $n_{\ell \alpha, i}$ near the edge, we can determine the boundary "spin" corresponding to each plot that strongly suggests the SPT state shown in the inset [18].

strong-coupling regime, persist down to realistic regions. Figure 2(a) shows the presence of exponentially localized edge states in the spin-resolved local densities $n_{\ell \alpha, i}$, which is a clear signature of the spin-Haldane (SH) phase with spin-1/2 edge states. The SPT phases for $N=3$ and 4 can also be probed using their particular edge states [Figs. 2(b) and 2(c)] or their entanglement spectra (ES) [Figs. 3(b) and 3(c)]. The precise nature of the edge states can be inferred from Fig. 2. For SU(3), we find that the phase for $t=t_{\perp}=1$ is a $\chi \mathrm{H}$ phase $\left(n_{\mathrm{Y}}(\mathcal{R}), n_{\mathrm{Y}}(\overline{\mathcal{R}})\right)=(1,2)$ with the left and right edge states, respectively, transforming in the $\mathbf{3}$ and $\overline{\mathbf{3}}$ representations of SU(3) [18]. As mentioned above, when the system is inversion-symmetric, this and the second $\chi \mathrm{H}$ phase $(2,1)$ must be degenerate; DMRG simulations randomly pick one of the two minimally entangled states. We can find the second one with slightly different initial parameters [18]. This signals the emergence of the $\chi \mathrm{H}$ phase $(1,2)$ or $(2,1)$ for $t=t_{\perp}=$ 1 , which spontaneously breaks inversion symmetry $[19,20]$. Similarly, for $N=4$, the edge states in Fig. 2(c) strongly suggest one of the three SPT phases $(2,2)$ protected by SU(4). The edge states belong to the self-conjugate antisymmetric representation of $\mathrm{SU}(4)$ with dimension 6, in agreement with previous studies $[17,44-46]$. To provide additional insight into these SPT phases, we plot in Fig. 3 their ES obtained by cutting the chain in the middle and computing the Schmidt eigenvalues of the ground-state wave function. The ES of the SH phase is known to exhibit double-degeneracy for all levels [11], which is a signature of the underlying SPT phase. Figure 3(a) shows the correct evenfold degeneracy in the low-lying part of the spectrum, giving further evidence for the SH phase. For higher $N$, we expect [46] that degeneracy must be compatible with $\mathrm{SU}(N)$ representations allowed for 

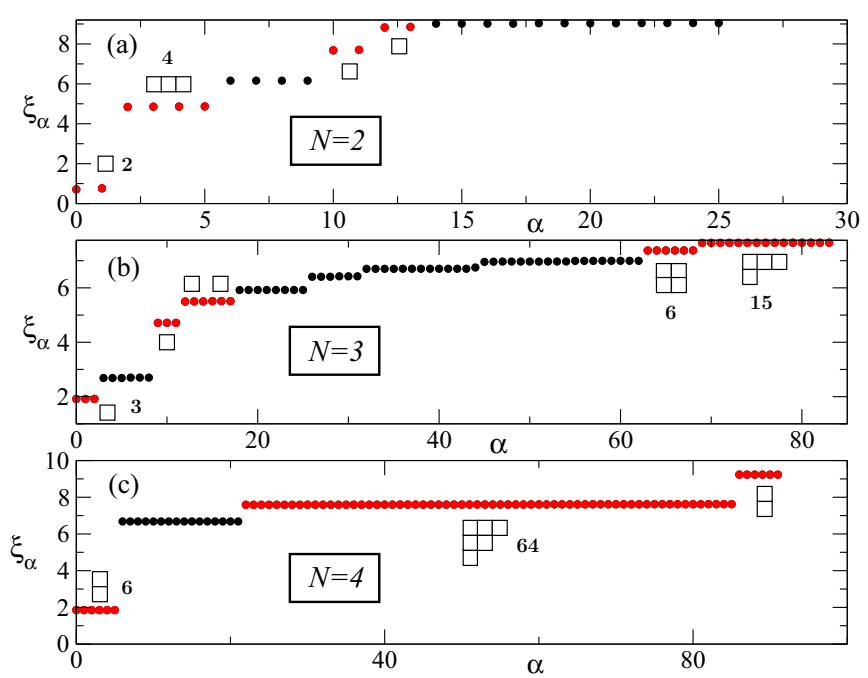

FIG. 3. The ES obtained by DMRG on the $L=48$ chain in the $N=2,3$, and 4 model (from top to bottom) using $t_{\perp}=1, U=20$, $V=2$. In all three cases, the system is in the SPT phase. Bosonic (fermionic) levels are shown by red (black) circles. Numbers and Young diagrams, respectively, denote the number of quasidegenerate levels and the corresponding edge representations. Since a representation cannot be distinguished from its conjugate by the degeneracy alone, we show [in (b)] only one of the two compatible with the assumed topological class.

the topological class (see Sec. IB of [18]). Since our ES are obtained for the fermionic model (4), some of the higher-lying levels belong to the "fermionic sector" of the spectrum and may not exhibit the structure expected in bosonic SPT phases (see, e.g., Refs. [50,51]). Hence, we separate the bosonic sector (shown by red circles) from the fermionic one (black circles) in Fig. 3. The degeneracy structure of the bosonic sector now perfectly agrees with what we expect for the corresponding SPT phases. Given the recent developments in entanglement measurements in cold-atom settings [52], our proposal would make the precise characterization of SPT phases possible in experiments. To show that the $\mathrm{SU}(N) \mathrm{SPT}$ phases found above are not restricted to the strong-coupling regime, we plot their extent in Fig. 4 as a function of $U$ along the physically relevant line $U / V=10$ at fixed $t_{\perp}=t(=1)$. These phases occur in the large- $U$ regime, and, for weaker interactions, quantum phase transitions are expected toward fully gapped trivial or dimerized phases, which break the translation symmetry spontaneously.

\section{SUMMARY AND EXPERIMENTAL PROSPECTS}

We have introduced a simple one-dimensional microscopic model describing alkali or alkaline-earth ultracold fermionic atoms loaded into a bichromatic optical lattice. Using analytical and numerical insight, we have shown how $\mathrm{SU}(N)$ SPT phases emerge for a large range of parameters. This provides a physical route to realize the SH phase $(N=$ 2 ), its generalization for even $N$, and the $\chi \mathrm{H}$ phase with $N=3$, which spontaneously breaks inversion symmetry. The SH phase with $N=2$ may be realized experimentally using the two lowest hyperfine states of ${ }^{6} \mathrm{Li}$, for which the ratio
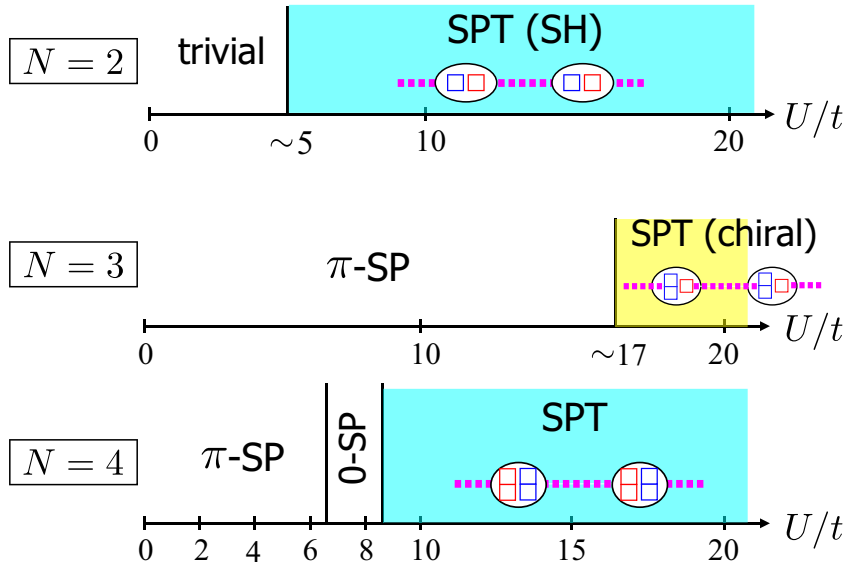

FIG. 4. Phase diagram for $N=2,3$, and 4 at fixed $t_{\perp}=t=1$, as a function of $U$ (with $U / V=10$; see the text) obtained from DMRG simulations. In all three cases, we find different SPT phases at strong coupling. For weaker interactions, we find trivial nondegenerate gapped phases, or the out-of-phase (in-phase) dimerized spin-Peierls-like $\pi$-SP (0-SP) phase.

$U / t$ may be tuned using a broad Feshbach resonance [53]. Furthermore, detection resolved in both density and spin is possible by combining a Fermi-gas microscope with SternGerlach techniques [54] or by ejecting unwanted spin states using resonant pulses $[55,56]$. The temperature scale of recent experiments involving ${ }^{6} \mathrm{Li}$ atoms is $T \simeq(0.5-0.8) 4 t^{2} / U$ [54]. This is of the same order of magnitude as the gap of the SH phase [49]: $\Delta_{\mathrm{SH}} \simeq 0.41 J \simeq 0.8 t^{2} / U$ for large $U$ [57]. As was recently shown numerically in Ref. [58], the main characteristics of the thermal spectral functions of the SH phase with localized edge states are still visible at finite size for $T \simeq \Delta_{\mathrm{SH}}$, a temperature scale that is within the reach of forthcoming experiments. Larger values of $N$ are experimentally accessible using fermionic alkaline-earth or ytterbium atoms. Using typical experimental values for ${ }^{173} \mathrm{Yb}$ ( $N=6$; scattering length $a_{g}=10.55 \mathrm{~nm}$ [59] and lattice spacing $\pi / k \approx 400 \mathrm{~nm}[60]$ ), we find $U / V \sim 10$. Spin-resolved measurements may be performed on these systems using optical Stern-Gerlach techniques $[61,62]$. Thanks to the recent experimental achievements with cold fermionic gases, we expect the SPT phases discussed in this paper to be observed in the near future.

\section{ACKNOWLEDGMENTS}

The authors are very grateful to V. Bois for his collaboration at the early stage of this work. We would like to thank G. Salomon for important discussions. The authors (S.C., P.L., and K.T.) are grateful to CNRS (France) for financial support (PICS grant). One of the authors (K.T.) is supported in part by JSPS KAKENHI Grants No. 15K05211, No. 18K03455, and No. JP15H05855. This work was performed using HPC resources from GENCI (Grants No. x2016050225 and No. A0010500225) and CALMIP. Last, the authors acknowledge the programs YITP-T-16-03 and YITP-T-17-01 held at Yukawa Institute for Theoretical Physics where parts of this work were carried out. 
[1] B. Zeng, X. Chen, D.-L. Zhou, and X.-G. Wen, arXiv:1508.02595.

[2] T. Senthil, Annu. Rev. Condens. Matter Phys. 6, 299 (2015).

[3] F. D. M. Haldane, Phys. Lett. A 93,464 (1983); Phys. Rev. Lett. 50, 1153 (1983).

[4] T. Kennedy, J. Phys.: Condens. Matter 2, 5737 (1990).

[5] M. Hagiwara, K. Katsumata, I. Affleck, B. I. Halperin, and J. P. Renard, Phys. Rev. Lett. 65, 3181 (1990).

[6] Z. C. Gu and X. G. Wen, Phys. Rev. B 80, 155131 (2009).

[7] F. Pollmann, E. Berg, A. M. Turner, and M. Oshikawa, Phys. Rev. B 85, 075125 (2012).

[8] X. Chen, Z.-C. Gu, and X.-G. Wen, Phys. Rev. B 83,035107 (2011); 84, 235128 (2011).

[9] N. Schuch, D. Perez-Garcia, and I. Cirac, Phys. Rev. B 84, 165139 (2011).

[10] L. Fidkowski and A. Kitaev, Phys. Rev. B 83, 075103 (2011).

[11] F. Pollmann, A. M. Turner, E. Berg, and M. Oshikawa, Phys. Rev. B 81, 064439 (2010).

[12] Z. Bi, A. Rasmussen, K. Slagle, and C. Xu, Phys. Rev. B 91, 134404 (2015).

[13] K. Duivenvoorden and T. Quella, Phys. Rev. B 87, 125145 (2013).

[14] As $\mathrm{SU}(N)$ does not possess nontrivial projective representations, we need to consider the projective unitary group $\mathrm{PSU}(\mathrm{N}) \cong \mathrm{SU}(\mathrm{N}) / \mathbb{Z}_{N}$ as the protecting symmetry.

[15] D. V. Else, S. D. Bartlett, and A. C. Doherty, Phys. Rev. B 88, 085114 (2013).

[16] K. Duivenvoorden and T. Quella, Phys. Rev. B 88, 125115 (2013)

[17] S. Capponi, P. Lecheminant, and K. Totsuka, Ann. Phys. 367, 50 (2016).

[18] See Supplemental Material at http://link.aps.org/supplemental/ 10.1103/PhysRevB.99.054414 for (i) an introduction to the representations of $\mathrm{SU}(N)$ and to topological phases, (ii) a discussion of the strong-coupling expansion for SU(3), and (iii) a detailed description of our numerical results.

[19] T. Morimoto, H. Ueda, T. Momoi, and A. Furusaki, Phys. Rev. B 90, 235111 (2014).

[20] S. Rachel, D. Schuricht, B. Scharfenberger, R. Thomale, and M. Greiter, J. Phys.: Conf. Ser. 200, 022049 (2010).

[21] I. Affleck, T. Kennedy, E. H. Lieb, and H. Tasaki, Commun. Math. Phys. 115, 477 (1988).

[22] A. Roy and T. Quella, Phys. Rev. B 97, 155148 (2018)

[23] H. Katsura, T. Hirano, and V. E. Korepin, J. Phys. A 41, 135304 (2008).

[24] M. A. Cazalilla, A. F. Ho, and M. Ueda, New J. Phys. 11, 103033 (2009).

[25] A. V. Gorshkov, M. Hermele, V. Gurarie, C. Xu, P. S. Julienne, J. Ye, P. Zoller, E. Demler, M. D. Lukin, and A. M. Rey, Nat. Phys. 6, 289 (2010).

[26] M. A. Cazalilla and A. M. Rey, Rep. Prog. Phys. 77, 124401 (2014).

[27] S. Taie, R. Yamazaki, S. Sugawa, and Y. Takahashi, Nat. Phys. 8, 825 (2012).

[28] G. Pagano, M. Mancini, G. Cappellini, P. Lombardi, F. Schafer, H. Hu, X.-J. Liu, J. Catani, C. Sias, M. Inguscio, and L. Fallani, Nat. Phys. 10, 198 (2014).

[29] X. Zhang, M. Bishof, S. L. Bromley, C. V. Kraus, M. S. Safronova, P. Zoller, A. M. Rey, and J. Ye, Science 345, 1467 (2014).
[30] F. Scazza, C. Hofrichter, M. Höfer, P. C. De Groot, I. Bloch, and S. Fölling, Nat. Phys. 10, 779 (2014).

[31] M. Atala, M. Aidelsburger, M. Lohse, J. T. Barreiro, B. Paredes, and I. Bloch, Nat. Phys. 10, 588 (2014).

[32] J. Sebby-Strabley, M. Anderlini, P. S. Jessen, and J. V. Porto, Phys. Rev. A 73, 033605 (2006).

[33] I. Bloch, J. Dalibard, and W. Zwerger, Rev. Mod. Phys. 80, 885 (2008).

[34] I. Bloch and M. Greiner, Adv. At. Mol. Opt. Phys. 52, 1 (2006).

[35] F. Werner, O. Parcollet, A. Georges, and S. R. Hassan, Phys. Rev. Lett. 95, 056401 (2005).

[36] More quantitatively, the energy associated with the densityassisted hopping terms is about 100 times smaller than $V$. This is so both for the ${ }^{173} \mathrm{Yb}$ parameters considered in the last paragraph of our paper and for the fermionic alkalis ${ }^{6} \mathrm{Li}$ and $40 \mathrm{~K}$.

[37] Typically, increasing $r$ makes, e.g., $t_{\perp} / t$ and $V / U$ smaller.

[38] C. Chin, R. Grimm, P. Julienne, and E. Tiesinga, Rev. Mod. Phys. 82, 1225 (2010).

[39] S. Taie, S. Watanabe, T. Ichinose, and Y. Takahashi, Phys. Rev. Lett. 116, 043202 (2016).

[40] K. Enomoto, K. Kasa, M. Kitagawa, and Y. Takahashi, Phys. Rev. Lett. 101, 203201 (2008).

[41] G. Cappellini, L. F. Livi, L. Franchi, D. Tusi, D. B. Orenes, M. Inguscio, J. Catani, and L. Fallani, Phys. Rev. X (to be published, 2019), arXiv:1810.09980.

[42] K. Kobayashi, M. Okumura, Y. Ota, S. Yamada, and M. Machida, Phys. Rev. Lett. 109, 235302 (2012).

[43] K. Kobayashi, Y. Ota, M. Okumura, S. Yamada, and M. Machida, Phys. Rev. A 89, 023625 (2014).

[44] V. Bois, S. Capponi, P. Lecheminant, M. Moliner, and K. Totsuka, Phys. Rev. B 91, 075121 (2015).

[45] H. Nonne, M. Moliner, S. Capponi, P. Lecheminant, and K. Totsuka, Europhys. Lett. 102, 37008 (2013).

[46] K. Tanimoto and K. Totsuka, arXiv:1508.07601.

[47] K. Wan, P. Nataf, and F. Mila, Phys. Rev. B 96, 115159 (2017).

[48] S. Capponi, P. Fromholz, P. Lecheminant, and K. Totsuka (unpublished).

[49] S. R. White, Phys. Rev. Lett. 69, 2863 (1992); U. Schollwöck, Rev. Mod. Phys. 77, 259 (2005).

[50] K. Hasebe and K. Totsuka, Phys. Rev. B 87, 045115 (2013).

[51] S. Moudgalya and F. Pollmann, Phys. Rev. B 91, 155128 (2015).

[52] R. Islam, R. Ma, P. M. Preiss, M. Eric Tai, A. Lukin, M. Rispoli, and M. Greiner, Nature (London) 528, 77 (2015).

[53] G. Zurn, T. Lompe, A. N. Wenz, S. Jochim, P. S. Julienne, and J. M. Hutson, Phys. Rev. Lett. 110, 135301 (2013).

[54] M. Boll, T. A. Hilker, G. Salomon, A. Omran, J. Nespolo, L. Pollet, I. Bloch, and C. Gross, Science 353, 1257 (2016).

[55] M. F. Parsons, A. Mazurenko, C. S. Chiu, G. Ji, D. Greif, and M. Greiner, Science 353, 1253 (2016).

[56] L. W. Cheuk, M. A. Nichols, K. R. Lawrence, M. Okan, H. Zhang, E. Khatami, N. Trivedi, T. Paiva, M. Rigol, and M. W. Zwierlein, Science 353, 1260 (2016).

[57] Note also that cooling should be more efficient for larger $N$ due to the Pomeranchuk effect; see, e.g., Z. Cai, H.-H. Hung, L. Wang, D. Zheng, and C. Wu, Phys. Rev. Lett. 110, 220401 (2013).

[58] J. Becker, T. Köhler, A. C. Tiegel, S. R. Manmana, S. Wessel, and A. Honecker, Phys. Rev. B 96, 060403(R) (2017). 
[59] M. Kitagawa, K. Enomoto, K. Kasa, Y. Takahashi, R. Ciurylo, P. Naidon, and P. S. Julienne, Phys. Rev. A 77, 012719 (2008).

[60] C. Hofrichter, L. Riegger, F. Scazza, M. Höfer, D. R. Fernandes, I. Bloch, and S. Fölling, Phys. Rev. X 6, 021030 (2016).
[61] S. Taie, Y. Takasu, S. Sugawa, R. Yamazaki, T. Tsujimoto, R. Murakami, and Y. Takahashi, Phys. Rev. Lett. 105, 190401 (2010).

[62] S. Stellmer, R. Grimm, and F. Schreck, Phys. Rev. A 84, 043611 (2011). 